\title{
Cognitive Aging in Dogs
}

\author{
Durga Chapagain Friederike Range Ludwig Huber Zsófia Virányi
}

Clever Dog Lab, Comparative Cognition, Messerli Research Institute, University of Veterinary Medicine, Vienna, Medical University of Vienna, University of Vienna, Vienna, Austria

\section{Keywords}

Pet dogs · Cognitive aging · Diagnosis · Prevention · Behavioral enrichment - Dietary intervention - Canine cognitive dysfunction

\begin{abstract}
A decline in the physical or mental health of older dogs can be a challenge for the owners, whose relationship with their dog is compromised by the cognitive and behavioral changes in their dogs. Although dog owners tend to consider many physiological and behavioral changes in old dogs as part of the normal aging process, it is important to differentiate between normal aging and pathologic aging, since behavioral changes may be the first indication of declining health and welfare in old dogs. Most reviews on cognitive aging in dogs have focused on translational approaches to human Alzheimer's disease; from a practical perspective, however, understanding normal cognitive aging in pet dogs and screening cognitively affected dogs are important in their own right. Here we review the literature on different cognitive functions that decline during aging, signs of cognitive dysfunction, screening methods, and preventive measures for age-related cognitive decline. Moreover, we discuss the drawbacks of using questionnaires as subjective measures of aging and propose the development of objective methods to distinguish normal cognitive aging from se-
\end{abstract}

\begin{tabular}{ll}
\hline KARGER & $\begin{array}{l}\text { ( ) 2017 The Author(s) } \\
\text { Published by S. Karger AG, Basel }\end{array}$ \\
$\begin{array}{l}\text { E-Mail karger@karger.com } \\
\text { www.karger.com/ger }\end{array}$ & $\begin{array}{l}\text { Karger } \\
\text { This article is licensed under the Creative Commons Attribution- } \\
\text { NonCommercial-NoDerivatives 4.0 International License (CC BY- } \\
\text { NC-ND) (http://www.karger.com/Services/OpenAccessLicense). } \\
\text { Usage and distribution for commercial purposes as well as any dis- } \\
\text { tribution of modified material requires written permission. }\end{array}$
\end{tabular}

vere cognitive dysfunction. We suggest that multi-targeted approaches that combine owner-evaluated questionnaires with neuropsychological tests can be most effective in screening cognitively affected dogs from normally aging dogs. Regarding preventive measures, we conclude that combinations of dietary intervention and behavioral enrichment may be more beneficial than single-pathway manipulations in delaying cognitive aging or retaining various cognitive functions during aging. (2017 The Author(s)

Published by S. Karger AG, Basel

\section{Background}

Aging is a multifactorial process that leads to a decline in the functions of most organs and tissues. Modern advances in technology, health care, and nutrition have led to a significant increase in the average life span of humans and animals. Recent animal demographic studies have shown that the population of old dogs is increasing, as is the incidence of degenerative changes associated with aging [1]. While most dog owners are informed about the physiological changes associated with aging, including changes in body composition, metabolic processes, and sensory abilities [2], many are not aware of the behavioral and cognitive changes associated with increasing age that may or may not result in severe cognitive dysfunction

Durga Chapagain, MSc

Comparative Cognition, Messerli Research Institute University of Veterinary Medicine, Vienna, Medical University of Vienna Veterinärplatz 1, AT-1210 Vienna (Austria)

E-Mail durgachapagain1@gmail.com 
[1]. Paradoxically, less is known about the changes associated with aging than the aging process itself. Most research on cognitive aging in dogs has focused on the translational approaches to human aging and Alzheimer's disease (AD) [see 3-6 for a review]; however, understanding normal cognitive aging in pet dogs and screening cognitively affected dogs are of utmost importance from a practical perspective. For this reason, we here discuss the literature on different cognitive functions that decline during aging, signs of cognitive dysfunction, screening methods for age-related cognitive decline, and different interventions used in pet dogs.

\section{Aging Processes}

Various theories have been put forth regarding the biological mechanisms that lead to aging, including free-radical-induced damages, molecular cross-linking, telomere shortening, and the presence of senescence genes in the DNA [7]. Most recently, a unified theory of aging has been proposed that encompasses major theories of aging [7]. The free-radical theory of aging provides explanations for the aging phenomena, including the connections between the average life spans of mammals and their basal metabolic rates [8], as well as between disease and aging [9]. The production of free radicals can lead to oxidative damage of proteins, lipids, and nucleotides that in turn may cause neuronal dysfunction and ultimately neuronal death [4]. Studies have highlighted that the level of oxidative stress plays a pivotal role in brain functioning, creating a delicate balance between free-radical production and brain protection or damage [10]. Oxidative damage progressively accumulates in the aging brain; damaged molecules can lead to impaired neuronal functions and vulnerability to the development of $\mathrm{AD}$. Brain aging then may lead to different degrees of behavioral and cognitive dysfunction [11]. Therefore, finding ways to minimize free-radical reaction damage in humans and animals could lead to a decrease in the morbidity and mortality due to degenerative changes associated with aging.

\section{The Dog as a Model for Human Aging}

Several studies have provided evidence that suggests that the dog is a good model system for human aging and is going to replace traditional rodent models and nonhuman primate models [3]. Dogs have been used to model health span and longevity, aging and associated diseases such as $\mathrm{AD}$ [12], and psychiatric disorders, such as human obsessive-compulsive disorder [13] and attention deficit hyperactivity disorder [14]. Most studies used laboratory beagles to model cognitive aging in humans and showed that in both species cognitive decline (detected in measures of learning, memory, attention, and executive functions) is associated with the accumulation of $\beta$-amyloid protein in senile plaques in the brain [5]. These findings suggest that age-related deficits in cognitive functions result from a similar neuropathology in dogs as well as in humans [5]. Furthermore, although aged beagles vary widely in their cognitive functioning, some do develop cognitive dysfunction syndrome (CDS) that is thought to be equivalent to humans' $\mathrm{AD}$, which gives a high translational value to this dog model. More recently, the focus has been slowly shifting from laboratory dogs to pet dogs because they share the human environment and lifestyle, are exposed to the same pollutants and risk factors of infections, and develop age-related diseases similar to those of humans [3].

\section{Normal Aging}

As in humans and all other animals, also in dogs different life stages are accompanied by different behavioral and cognitive characteristics, including changes in playfulness or in being able to concentrate and learn $[6,15]$. The cognitive changes aging humans go through are relatively well studied and, importantly, they are thought to be adaptive as they ensure maintenance or even improvement of the well-being of old individuals [16]. Much less is known about the life span cognition of dogs. Research in laboratory dogs, similarly to humans, has reported a decline in learning, memory, and executive control with increasing age $[5,17-19]$, and a few recent studies in pet dogs have investigated how social responsiveness, curiosity towards novel objects, attentiveness, learning, and memory change with increasing age [20-23]. Considering such lifelong changes in behavior and cognition, the question arises of when aging begins in dogs. As there is a huge variation in how long dogs of different breeds live [24], the point when aging begins varies with the breed, size, and weight of the dog [25]. Different authors have made different suggestions in this regard (Golini et al. [26] have reported 7 years, while Neilson et al. [27] have indicated 11 years). A majority of aging studies in laboratory beagles have compared dogs aged over 7 years to younger dogs (age $<7$ years) in order to investigate cognitive decline associated with aging. However, beagles can 
show short-term memory impairments as early as 6 years of age [28]. Furthermore, different cognitive functions have been proposed to follow different developmental trajectories, which likely means that they also decline at different ages and rates. Importantly, these aging trajectories differ from one individual to another [5]. Some dogs age in a way that may not directly affect their dayto-day functioning as pets or their activities and relationship with their owners, while for others their decreasing performance may compromise these functions. Monitoring the cognitive and behavioral aging of service dogs, working dogs, or sports dog may be very important even in successfully aging individuals, as their performance efficiency may be affected while they age. Salvin et al. [29] reported that some old dogs (aged $\geq 8$ years and of different breeds), even if aging normally, might encounter fast age-related deterioration in activity, play, and response to commands, and an increase in fears and phobias within a 6-month period [29]. Another part of the aging dog population develops cognitive impairment and dementia similar to neuropathological aging in humans $[1,30]$. Therefore, it is important to differentiate between normal cognitive aging and pathologic brain aging. Behavioral changes may be the first indication of declining health and welfare of old dogs and might help to understand whether the animal is affected by normal or pathologic aging [1]. Veterinarians and, ideally, even owners should be informed of what constitutes a normal decline of various cognitive functions in order to know how to live with aging dogs. It is also important to recognize signs of cognitive decline that begin too early (age $<6$ years) or proceed in an accelerated manner. Medical intervention and other techniques, if applied early enough, can help to slow its progress.

\section{Pathological Aging: Symptoms, Relevance, and Prevalence}

The neurobehavioral syndrome that may affect dogs over 8 years of age is usually referred to as canine cognitive dysfunction or CDS. It is characterized by progressive neurodegenerative changes in the cerebral cortex and the hippocampus that lead to a decline in cognitive functions and are clinically expressed as distinctive changes in the behavior and daily routines of dogs [1,31]. Dogs with CDS develop a neuropathology that includes $A \beta$ pathology, a reduced brain volume, neuronal loss, and impaired neurogenesis, like humans with $\mathrm{AD}$. The clinical signs displayed by affected dogs can be summarized by the ac-

Cognitive Aging in Dogs ronym DISHA, which refers to disorientation, a decrease in social interactions, changes in sleep-wake cycles, a loss of prior housetraining, increased anxiety, and changes in their level of activity [1]. Additionally, a decrease in the ability to find dropped food has been reported for dogs diagnosed with CDS [32]. Dogs with cognitive dysfunction show a substantial cognitive decline in tests measuring spatial learning and memory [28]. Moreover, CDS dogs are also reported to display erratic locomotion patterns, an increased frequency of aimless behaviors, and changes in social responsiveness such as a decreased response to both social isolation and human interaction $[15,22]$. Overall, changes associated with CDS may affect the dog-human bond as dogs may become less capable to communicate and interact with their owner.

Interestingly, it is rather unclear to what extent the pet dog population is affected by CDS. This is partly due to the high degree of variability in how dogs age and to the lack of systematic CDS evaluation criteria. The prevalence rate of CDS varies depending on the behaviors (disorientation, interaction changes, sleep/wake disturbances, house soiling and activity changes, anxiety and olfaction, learning and memory, etc.) that are taken into account. For example, Neilson et al. [27] reported that $28 \%$ of dogs aged 11-12 years had impairments in 1 or more categories of behavior, and $10 \%$ of them had impairments in 2 or more categories. In contrast, $68 \%$ of dogs aged 15-16 years had impairments in 1 or more categories, and $35 \%$ of them had impairments in 2 or more categories. Recently, Salvin et al. [32] developed a CDS rating scale for the diagnosis of cognitive dysfunction and reported the prevalence to be $14.2 \%$ in pet dogs over the age of 8 years in contrast to the veterinary diagnosis rate of $1.9 \%$. Overall, when including different studies, the prevalence of CDS in the population of senescent dogs ( $\geq 8$ years) is estimated to range from 14.2 to $22.5 \%$ and to increase exponentially with increasing age [27, 33-35].

\section{Tools to Diagnose Cognitive Aging in Dogs}

The diagnosis of cognitive aging (either normal aging or CDS) in dogs is hindered by the lack of well-established diagnostic tools. Currently, veterinarians rely on the owners' assessment of their dog's behavior; they use observational screening questionnaires targeting the behavioral changes related to normal cognitive aging or cognitive dysfunction. Several CDS questionnaires or scales have been developed $[1,32,36-40]$, but unfortunately the design and scoring methods are diverse. Moreover, it is

Gerontology 2018;64:165-171 167 
somewhat arbitrary how different behavioral items are selected within the above listed domains of DISHA, categorized and scored in order to obtain a total cognitive score. Therefore, systematic evaluation criteria for diagnosing CDS are missing, and the information on the diagnostic sensitivity, reliability, validity, and comparability of the existing questionnaires is scant [25]. Some studies, like those of Rofina et al. [36] and Schütt et al. [41], have tried to compare the already existing CDS questionnaires and documented that the total questionnaire scores correlated well between the questionnaires within the group of CDS dogs; however, no correlation was found between the questionnaire scores within the group classified as successfully aging dogs. Therefore, these studies suggest that the questionnaires developed by Salvin et al. [32] and Rofina et al. [36] are capable of screening only severe cognitive dysfunction dogs from a population of aged pet dogs. Detection of mild impairment, however, would be necessary to start a medical intervention in time. As an alternative, Madari et al. [42] developed the Canine Dementia Scale in order to identify various stages of cognitive impairment, ranging from mild through moderate cognitive impairments to severe cognitive dysfunction.

In contrast, Adams et al. [5] suggested a more objective way of detecting cognitive aging in laboratory dogs on the basis of their performance in neuropsychological tests. These researchers used a modified Wisconsin General Testing Apparatus, securing motor and sensory demands of dogs across tests. Aged laboratory dogs showed cognitive decline in tests measuring complex learning, executive function, spatial learning and memory [18, 28, 43]. Using spatial learning and memory tasks (Delayed Nonmatch to Position (DNMP)), old dogs can be categorized into 3 groups: successful agers, dogs with mild cognitive impairments, and severely impaired dogs [5]. Since the tasks were developed in laboratory beagles, they require an extensive pre-training protocol and a substantial amount of time to finish the training and to complete the task. Recently, such methods have been used in pet dogs as well [23]. Wallis et al. [23] used visual discrimination tasks ( 4 vs. 4 picture discrimination task) to measure learning and memory in a cohort of pet Border collies aged 6 months to 14 years and documented that learning decreases with increased age. However, using a similar task ( 2 vs. 2 picture discrimination task), Chapagain et al. [unpubl. data] reported no influence of age on the learning speed of pet dogs of different breeds aged over 6 years. To fulfill the learning criteria in these tasks, dogs took a long time (several months). Consequently, these tests are not suitable for a veterinary diagnosis of cognitive aging in pet dogs in clinical settings.

Other tests like open field tests measuring behavior associated with locomotion, exploration and social responsiveness [15, 22], and food-seeking [44], or place selection tests [45] and tests to assess spatial reversal learning [46], have more recently been introduced in pet dogs to measure cognitive decline. Rosado et al. $[15,22]$ assessed the influence of age and severity of CDS on locomotion and exploratory behavior, and social responsiveness in pet dogs. They reported that the severity of CDS influenced locomotion and exploratory behavior. In particular, they found that the more severe their impairment was, the more locomotion and corner-directed (aimless) behaviors they showed, and the lower their frequency of doordirected activities was. Moreover, severe CDS dogs displayed more sniffing episodes directed towards the objects. Changes in social responsiveness associated with severe CDS included a decreased response to social isolation and human interaction, and increased time spent in front of the mirror, suggesting a deficit in habituation. González-Martínez et al. [44] evaluated the effect of age and severity of cognitive dysfunction on 2 simple tasks (a food-searching task and a problem-solving task) and reported that dogs with severe CDS displayed a poor performance in retrieving food compared to mild CDS dogs or cognitively unimpaired aged dogs. In the problemsolving task, younger dogs outperformed older dogs; however, no differences related to CDS severity were found. Therefore, the results of these 3 studies highlight the testing of spontaneous activity and food-seeking abilities to better characterize CDS in aged pet dogs. However, in these studies, owner-reported questionnaires were used to characterize the severity of cognitive dysfunction. In a simple place selection task, Nagasawa et al. [45] found that the learning speed of the dogs decreased as they aged. They reported, however, no correlation with regard to the severity of the cognitive decline. Mongillo et al. [46] found that spatial reversal learning assessed using a $T$-maze was impaired in pet dogs aged over 8 years compared to dogs younger than 8 years. This study highlighted the effects of aging on the processing speed and executive function of dogs; however, it again reported no comparison with respect to the severity of cognitive decline. Probably for this reason, these tests so far have contributed only to basic research but they have not been used by veterinarians in clinical settings. More recently, using 145 Border collies aged 6 months to 14 years, different tests measuring attention, learning and memory were developed and tested by our research group [20,23]. In Bor- 
der collies, these tests proved suitable to detect aging effects on attentiveness and learning [20,23], and these age effects were then confirmed in another study where aged pet dogs of various breeds showed a decline in attention [21]. Extending on these tests, we developed a test battery measuring different cognitive functions, such as learning and trainability, social and physical cognition, attentiveness, food and toy motivation, problem-solving ability, inhibition, memory and persistency, sociability, and exploration and dependency. This so-called Vienna Canine Cognitive Battery aims to measure cognitive aging in pet dogs. As the test battery can be conducted in a short time (1-2 h) and its use is feasible also in general veterinary practice, we hope to provide a useful tool to diagnose cognitive dysfunction in pet dogs in clinical settings by examining correlated individual differences in the domains of general, social, and physical cognition as well as human-animal interactions.

\section{Interventions in Dogs}

Not only has cognitive aging been demonstrated in laboratory beagle dogs [47] but also interventions that improve cognition or delay cognitive decline have been tested in such populations [48]. The possible treatments for cognitive impairment include alleviation of the damaging effects of free radicles produced by oxidative stress during aging and enhancing mitochondrial function [47]. Landsberg et al. [1] presented a list of drugs that may be considered to improve cognitive function or control the clinical signs of CDS. Specifically, medications such as selegiline and propentofylline [49] are prescribed for the treatment of CDS when the symptoms are more pronounced. However, providing dietary supplements containing cellular antioxidants (vitamins $\mathrm{C}$ and $\mathrm{E}$ ), mitochondrial cofactors (lipoic acid and carnitine), and omega-3 fatty acids at an early age (around 6 years) minimize the risk factors associated with brain damage caused by CDS [49]. This has been demonstrated by studies where dogs fed the supplemented diet showed reduced oxidative damage and mitochondrial dysfunction and a significant reduction in $\beta$-amyloid pathology across multiple brain regions compared to dogs fed a control diet $[12,50]$. Aged dogs fed a diet containing medium-chain triglyceride supplementation performed better on various cognitive tasks [51]. Behavioral enrichment (physical exercise, social enrichment, and cognitive enrichment), alone or in combination with an antioxidant diet, also preserves and improves cognitive function in old dogs $[43,52]$. Since

Cognitive Aging in Dogs these data have almost exclusively been collected under laboratory conditions and in beagles that were mostly obtained from highly inbred lines and had limited social and environmental experiences, currently we do not know if behavioral enrichment and nutritional treatment have similar effects on pet dogs that live in a physically and socially more stimulating environment posing more cognitive challenges than the life of laboratory dogs. Such studies testing preventive approaches to CDS on pet dog populations are very important as they have a high practical relevance for the welfare of dogs and their life with their owners.

So far, one study has shown that lifelong training can delay the aging of attentional capture, sustained attention, and selective attention in pet dogs of various breeds [21], and two studies have looked at the effect of nutritional supplementation on pet dogs exhibiting symptoms of cognitive dysfunction. Heath et al. [53] investigated the use of "Aktivait" containing a range of antioxidants, freeradical scavengers, vitamins $\mathrm{C}$ and $\mathrm{E}$, fatty acids, and phosphatidylserine in 44 pet dogs of different breeds for 42 days. They monitored the behavior of the dogs for 56 days via questionnaires and reported that there was a significant improvement in the dogs' behavior as noted by the owners during the trial period. However, the authors suggested that a larger sample size and a longer feeding duration could make the results more robust. Also using questionnaire data, Dodd et al. [54] looked at the effect of feeding fortified food to old pet dogs for 60 days and concluded that those dogs showed significant improvement in different behavioral categories compared to control groups. These studies investigated dietary effects over shorter time periods and relied exclusively on the owners' assessment of their dogs' behavior. Such a subjective rating of the behavior of the dogs may either mask or enlarge some effects that could have been assessed more objectively by testing the dogs in different cognitive tests. Also, the behavior of a larger sample of dogs including multiple breeds might be more representative of the pet dog population.

\section{Conclusion}

We have briefly outlined the importance of early recognition of signs of cognitive aging and in particular cognitive dysfunction in aged pet dogs to enhance the health and welfare of dogs and retain the dog-owner bond. Since owner-evaluated questionnaires may be insensitive to early and subtle changes in learning and memory and

Gerontology 2018;64:165-171 DOI: $10.1159 / 000481621$ 
other specific cognitive functions associated with brain aging in dogs, an alternative method is needed that involves a battery of neuropsychological tests and provides measures of various cognitive functions. Neuropsychological tests are needed that can be used to objectively assess cognitive changes as a function of age in dogs, to characterize the biological basis of age-dependent cognitive decline, and to test potential therapeutic interventions, including dietary treatment. Nonetheless, certain questionnaires like that of Salvin et al. [32] seem to be able to detect dogs that are already showing signs of severe cognitive dysfunction, while a questionnaire developed by Madari et al. [42] could identify various stages of cognitive impairment. Therefore, a combination of different approaches, such as owner-evaluated questionnaires and neuropsychological tests, may be the most effective in differentiating cognitive dysfunction dogs from normally aging dogs. Additionally, a combination of dietary intervention and behavioral enrichment may be more beneficial than single-pathway manipulations. Most reviews on cognitive aging in dogs have so far focused on translational approaches to human $\mathrm{AD}$. Here, our aim was to call attention to the fact that screening cognitively affected dogs and providing appropriate interventions to delay cognitive aging or to retain cognitive functions have an enormous impact on the well-being of aging dogs. This in turn has an effect on the everyday life of dog owners and may provide dog owners with years of quality time with their beloved companions.

\section{Acknowledgements}

We would like to thank Julia Schoesswender, Lisa J. Wallis, Manuel Kemethofer, and Karin Bayer for their help in the Dog Aging Project.

\section{Funding Sources}

The work in this paper and the research on dog aging in Vienna were fully funded by Royal Canin, Aimargues, France (project No. FA 3721 4007).

\section{Author Contributions}

D.C., Z.V., F.R., and L.H. conceived this review. D.C. prepared the first draft of this paper. D.C., Z.V., F.R., and L.H. revised this paper.

\section{References}

1 Landsberg GM, Nichol J, Araujo JA: Cognitive dysfunction syndrome: a disease of canine and feline brain aging. Vet Clin North Am Small Anim Pract 2012;42:749-768, vii.

2 Larsen JA, Farcas A: Nutrition of aging dogs. Vet Clin North Am Small Anim Pract 2014; 44:741-759.

3 Kaeberlein M, Creevy KE, Promislow DEL: The Dog Aging Project: translational geroscience in companion animals. Mamm Genome 2016;27:279-288.

4 Head E: A canine model of human aging and Alzheimer's disease. Biochim Biophys Acta 2013;1832:1384-1389.

5 Adams B, Chan A, Callahan H, Milgram NW: The canine as a model of human cognitive aging: recent developments. Prog Neuropsychopharmacol Biol Psychiatry 2000;24:675692.

6 Davis PR, Head E: Prevention approaches in a preclinical canine model of Alzheimer's disease: benefits and challenges. Front Pharmacol 2014;5:47.

7 da Costa JP, Vitorino R, Silva GM, Vogel C, Duarte AC, Rocha-Santos T: A synopsis on aging: theories, mechanisms and future prospects. Ageing Res Rev 2016;29:90-112.

8 Harman D: The aging process. Proc Natl Acad Sci USA 1981;78:7124-7128.
9 Harman D: The aging process: major risk factor for disease and death. Proc Natl Acad Sci USA 1991;88:5360-5363.

10 Chakrabarti S, Munshi S, Banerjee K, Thakurta IG, Sinha M, Bagh MB: Mitochondrial dysfunction during brain aging: role of oxidative stress and modulation by antioxidant supplementation. Aging Dis 2011;2:242-256.

11 Youssef SA, Capucchio MT, Rofina JE, Chambers JK, Uchida K, Nakayama H, et al: Pathology of the aging brain in domestic and laboratory animals, and animal models of human neurodegenerative diseases. Vet Pathol 2016; 53:327-348.

12 Opii WO, Joshi G, Head E, Milgram NW, Muggenburg BA, Klein JB, et al: Proteomic identification of brain proteins in the canine model of human aging following a long-term treatment with antioxidants and a program of behavioral enrichment: relevance to Alzheimer's disease. Neurobiol Aging 2008;29:51-70.

13 Rapoport JL, Ryland DH, Kriete M: Drug treatment of canine acral lick: an animal model of obsessive-compulsive disorder. Arch Gen Psychiatry 1992;49:517-521.

14 Lit L, Schweitzer JB, Iosif A-M, Oberbauer AM: Owner reports of attention, activity, and impulsivity in dogs: a replication study. Behav Brain Funct 2010;6:1.
15 Rosado B, González-Martínez Á, Pesini P, García-Belenguer S, Palacio J, Villegas A, et al: Effect of age and severity of cognitive dysfunction on spontaneous activity in pet dogs. 1. Locomotor and exploratory behaviour. Vet J 2012;194:189-195.

16 Carstensen LL, Mikels JA, Mather M: Aging and the intersection of cognition, motivation and emotion; in Birren J, Schaie KW (eds): Handbook of the Psychology of Aging. Amsterdam, Elsevier, pp 343-362.

17 Milgram NW, Head E, Weiner E, Thomas E: Cognitive functions and aging in the dog: acquisition of nonspatial visual tasks. Behav Neurosci 1994;108:57-68.

18 Tapp PD, Siwak CT, Estrada J, Holowachuk D, Milgram NW: Effects of age on measures of complex working memory span in the beagle dog (Canis familiaris) using two versions of a spatial list learning paradigm. Learn Mem 2003;10:148-160.

19 Tapp PD, Siwak CT, Estrada J, Head E, Muggenburg BA, Cotman CW, et al: Size and reversal learning in the beagle dog as a measure of executive function and inhibitory control in aging. Learn Mem 2003;10:64-73. 
20 Wallis L, Range F, Müller C, Serisier S, Huber L, Virányi Z: Lifespan development of attentiveness in domestic dogs: drawing parallels with humans. Front Psychol 2014;5:71.

21 Chapagain D, Virányi Z, Wallis LJ, Huber L, Serra J, Range F: Aging of attentiveness in border collies and other pet dog breeds: the protective benefits of lifelong training. Front Aging Neurosci 2017;9:100.

22 Rosado B, González-Martínez A, Pesini P, García-Belenguer S, Palacio J, Villegas A, et al: Effect of age and severity of cognitive dysfunction on spontaneous activity in pet dogs. 2. Social responsiveness. Vet J 2012;194:189195.

23 Wallis LJ, Viranyi Z, Müller CA, Serisier S, Huber L, Range F: Aging effects on discrimination learning, logical reasoning and memory in pet dogs. Age 2016;38:6.

24 O'Neill DG, Church DB, McGreevy PD, Thomson PC, Brodbelt DC: Longevity and mortality of owned dogs in England. Vet J 2013;198:638-643.

25 Szabó D, Gee NR, Miklósi Á: Natural or pathologic? Discrepancies in the study of behavioral and cognitive signs in aging family dogs. J Vet Behav Clin Appl Res 2016;11:8698.

26 Golini L, Colangeli R, Tranquillo V, Mariscoli M: Association between neurologic and cognitive dysfunction signs in a sample of aging dogs. J Vet Behav Clin Appl Res 2009;4: 25-30.

27 Neilson JC, Hart BL, Cliff KD, Ruehl WW: Prevalence of behavioral changes associated with age-related cognitive impairment in dogs. J Am Vet Med Assoc 2001;218:17871791.

28 Studzinski CM, Christie L-A, Araujo JA, Burnham WM, Head E, Cotman CW, et al: Visuospatial function in the beagle dog: an early marker of cognitive decline in a model of human aging and dementia. Neurobiol Learn Mem 2006;86:197-204.

29 Salvin HE, McGreevy PD, Sachdev PS, Valenzuela MJ: Growing old gracefully - behavioral changes associated with "successful aging" in the dog, Canis familiaris. J Vet Behav Clin Appl Res 2011;6:313-320.

30 Siwak CT, Tapp PD, Head E, Zicker SC, Murphey HL, Muggenburg BA, et al: Chronic antioxidant and mitochondrial cofactor administration improves discrimination learning in aged but not young dogs. Prog Neuropsychopharmacology Biol Psychiatry 2005;29:461469.

31 Fast R, Schütt T, Toft N, Møller A, Berendt M: An observational study with long-term follow-up of canine cognitive dysfunction: clinical characteristics, survival, and risk factors. J Vet Intern Med 2013;27:822-829.
32 Salvin HE, McGreevy PD, Sachdev PS, Valenzuela MJ: The canine cognitive dysfunction rating scale (CCDR): a data-driven and ecologically relevant assessment tool. Vet J 2011; 188:331-336

33 Osella MC, Re G, Odore R, Girardi C, Badino $\mathrm{P}$, Barbero R, et al: Canine cognitive dysfunction syndrome: prevalence, clinical signs and treatment with a neuroprotective nutraceutical. Appl Anim Behav Sci 2007;105:297-310.

34 Azkona G, García-Belenguer S, Chacón G, Rosado B, León M, Palacio J: Prevalence and risk factors of behavioural changes associated with age-related cognitive impairment in geriatric dogs. J Small Anim Pract 2009;50:8791.

35 Salvin HE, McGreevy PD, Sachdev PS, Valenzuela MJ: Under diagnosis of canine cognitive dysfunction: a cross-sectional survey of older companion dogs. Vet J 2010;184:277-281.

36 Rofina JE, van Ederen AM, Toussaint MJM, Secrève M, van der Spek A, van der Meer I, et al: Cognitive disturbances in old dogs suffering from the canine counterpart of Alzheimer's disease. Brain Res 2006;1069:216-226.

37 Yu C-H, Song G-S, Yhee J-Y, Kim J-H, Im K-S, Nho W-G, et al: Histopathological and immunohistochemical comparison of the brain of human patients with Alzheimer's disease and the brain of aged dogs with cognitive dysfunction. J Comp Pathol 2011;145:45-58.

38 González-Martínez Á, Rosado B, Pesini P, Suárez M-L, Santamarina G, García-Belenguer $S$, et al: Plasma $\beta$-amyloid peptides in canine aging and cognitive dysfunction as a model of Alzheimer's disease. Exp Gerontol 2011;46:590-596.

39 Kiatipattanasakul W, Nakamura S, Hossain MM, Nakayama H, Uchino T, Shumiya S, et al: Apoptosis in the aged dog brain. Acta Neuropathol 1996;92:242-248.

40 Colle M-A, Hauw J-J, Crespeau F, Uchihara T, Akiyama H, Checler F, et al: Vascular and parenchymal $\mathrm{A} \beta$ deposition in the aging dog: correlation with behavior. Neurobiol Aging 2000;21:695-704.

41 Schütt T, Toft N, Berendt M: A comparison of 2 screening questionnaires for clinical assessment of canine cognitive dysfunction. J Vet Behav Clin Appl Res 2015;10:452-458.

42 Madari A, Farbakova J, Katina S, Smolek T, Novak P, Weissova T, et al: Assessment of severity and progression of canine cognitive dysfunction syndrome using the CAnine DEmentia Scale (CADES). Appl Anim Behav Sci 2015;171:138-145.
43 Milgram NW, Head E, Zicker SC, IkedaDouglas CJ, Murphey H, Muggenburg B, et al: Learning ability in aged beagle dogs is preserved by behavioral enrichment and dietary fortification: a two-year longitudinal study. Neurobiol Aging 2005;26:77-90.

44 González-Martínez A, Rosado B, Pesini P, García-Belenguer S, Palacio J, Villegas A, et al: Effect of age and severity of cognitive dysfunction on two simple tasks in pet dogs. Vet J 2013;198:176-181.

45 Nagasawa M, Yatsuzuka A, Mogi K, Kikusui T: A new behavioral test for detecting decline of age-related cognitive ability in dogs. J Vet Behav Clin Appl Res 2012;7:220-224.

46 Mongillo P, Araujo JA, Pitteri E, Carnier P, Adamelli S, Regolin L, et al: Spatial reversal learning is impaired by age in pet dogs. Age 2013;35:2273-2282.

47 Milgram NW, Zicker SC, Head E, Muggenburg BA, Murphey H, Ikeda-Douglas CJ, et al: Dietary enrichment counteracts age-associated cognitive dysfunction in canines. Neurobiol Aging 2002;23:737-745.

48 Fahnestock M, Marchese M, Head E, Pop V, Michalski B, Milgram WN, et al: BDNF increases with behavioural enrichment and an antioxidant diet in the aged dog. Neurobiol Aging 2012;33:546-554.

49 Landsberg GM, Deporter T, Araujo JA: Clinical signs and management of anxiety, sleeplessness, and cognitive dysfunction in the senior pet. Vet Clin North Am Small Anim Pract 2011;41:565-590.

50 Pop V, Head E, Hill M-A, Gillen D, Berchtold NC, Muggenburg B, et al: Synergistic effects of long-term antioxidant diet and behavioral enrichment on beta-amyloid load and nonamyloidogenic processing in aged canines. J Neurosci 2010;30:9831-9839.

51 Pan Y, Larson B, Araujo JA, Lau W, de Rivera C, Santana R, et al: Dietary supplementation with medium-chain TAG has long-lasting cognition-enhancing effects in aged dogs. $\mathrm{Br}$ J Nutr 2010;103:1746-1754.

52 Milgram NW, Head E, Zicker SC, IkedaDouglas C, Murphey H, Muggenberg BA, et al: Long-term treatment with antioxidants and a program of behavioral enrichment reduces age-dependent impairment in discrimination and reversal learning in beagle dogs. Exp Gerontol 2004;39:753-765.

53 Heath SE, Barabas S, Craze PG: Nutritional supplementation in cases of canine cognitive dysfunction: a clinical trial. Appl Anim Behav Sci 2007;105:284-296.

54 Dodd CE, Zicker SC, de Jewell: Can a fortified food affect the behavioral manifestations of age-related cognitive decline in dogs? Vet Med Anim Clin 2003;98:396. 